\title{
1. Project Title: Understanding the Fundamental Properties of Dark Matter and Dark Energy in Structure Formation and Cosmology
}

DOE Grant Number: DE-SC0001101

Principal Investigator: Dr. Richard S Ellis, California Institute of Technology

1200 E. California Blvd, MC 249-17, Pasadena, CA 91125,

Tel: 626-395-2598, email: rse@,astro.caltech.edu

Report for the period: July 12009 through June 302012 (Final Report)

Remaining Funds: Nil at the end of the current budget period

\section{Report for award ending June 302012 (Final Report)}

The program was concerned with developing and verifying the validity of observational methods for constraining the properties of dark matter and dark energy in the Universe.

Excellent progress has been made in measuring the properties of dark energy through the use of distant Type Ia supernova ( $\mathrm{SNe}$ ). The most significant milestone in this area was analysis of the third year Supernova Legacy Survey data in which the PI played a key spectroscopic role. The resulting constraints on the equation of state parameter, $w$, are the most precise so far obtained by any method. Moreover, analysis of the systematic errors associated with this program have highlighted the importance of understanding variations in the properties of $\mathrm{SNe}$ as a function of redshift, progenitor composition and host galaxy environment. The PI has led global efforts to evaluate these variations both through statistical studies of the ultraviolet spectroscopy of local $\mathrm{SNe}$ with the Palomar Transient Factory and Hubble Space Telescope and through detailed modeling of individual events where the physical origin of the variations are now understood.

The program also led to new constraints on the distribution of dark matter on small scales $(<100$ $\mathrm{kpc})$ via precise measures of its spatial distribution in clusters of galaxies using gravitational lensing. Although the 'cold' dark matter (CDM) model has been successful in explaining the large scale distribution of material, only on smaller scales can variants such as 'warm' dark matter be tested. In recent years, a number of discrepancies have arisen between observations and $\mathrm{CDM}$ on these scales, one of the most significant of which is the relative distribution of baryons and dark matter in clusters and dwarf galaxies. Via this program we have completed the first comprehensive study of the 3-D distribution of dark matter in clusters for comparison with numerical models and a key test of the claimed universal Navarro, Frenk and White (NFW) dark matter profile. Our work has demonstrated significant deficiencies in our understanding of dark matter and the likely importance of early interactions between baryons on small scales.

Weak gravitational lensing signals also offer a promising probe of dark energy. Via this program, Caltech led various studies to test the limiting effects in this approach. Subaru imaging data was followed up with Keck spectroscopy to examine the difficulties of counting clusters, selected via their lensing signals, as a probe of dark energy. This was paralleled by technical studies to improve algorithms for extracting the weak lensing signals as well as the effect of imperfections in current optical and near-infrared detectors.

The grant provided partial support for one postdoctoral researcher Dr Jeff Cooke who worked on the supernova program, and a full-time graduate student Drew Newman who worked on the dark matter program. Two undergraduates Mike Maseda and Sedona Price were involved in the Caltech program. Strong collaborative programs were enabled with JPL (Rhodes), LBNL (Nugent), Oklahoma (Baron), Mazzali (Max Planck), Weizman Institute (Gal-Yam), Harvard (Sand), UC Santa Barbara (Treu, Howell, Sand), Oxford (Sullivan) and Smith (Birmingham). 
The overall program led to 25 scientific papers which appeared or have been submitted to refereed journals.

More details of the accomplishments are given below.

\subsection{Supernovae as a Probe of Dark Energy}

Supernovae of Type Ia (SNe Ia) represent the most well-explored tool for probing dark energy. The program saw completion of our analysis of the first three years of data from the SuperNova Legacy Survey (SNLS) which has delivered the best measure to date for the equation of state of dark energy, $w=-1.061 \pm 0.068$ (combined with WMAP7 and for a flat Universe), based on detailed spectroscopic and photometric data of 472 distant SNe. The constraints on possible evolution in the equation of state are also impressive. For the formalism $w(a)=w_{0}+w_{a}(1-a)$, we find $w_{0}=0.905 \pm 0.196 ; w_{a}=-0.984 \pm 1.09$ in a flat Universe (combining in this case with WMAP7, SDSS and including a prior on the Hubble constant). In summary, our work provides the best evidence to date for dark energy with an equation of state parameter $w=-1$. The PI's contribution to this program were fundamental and included spectroscopic determinations of the SN type and redshift and studies of the host galaxy properties.

An important component of our achievements is a rigorous assessment of the systematic errors involved and the prospects for improving these constraints in the near future, e.g. with WFIRST and LSST. Through this grant the PI has played a leading role in these investigations. Given SNe are events that occur in evolving stellar populations, a key issue is whether there is some form of 'systematic floor' in their calibration and application, arising perhaps from time evolution in their behavior or intrinsic dispersion in their properties. The focus of this effort has been a careful examination of the rest-frame ultraviolet (UV) spectra which act as a valuable proxy for the progenitor metallicity - a key variable. Through a concerted campaign with the Palomar Transient Factory (PTF) and the STIS UV spectrograph, we completed a study of the maximum light spectra for 30 local events. This represents a 10-fold improvement in the sample size of nearby SNe with high quality UV spectra. This work has provided definitive evidence that the mean SN Ia spectrum is stable over the redshift range $0<\mathrm{z}<0.5$ but that the dispersion we found in Keck data at $\mathrm{z} \sim 0.5$ in 2008 is also seen in the local data. The spectral dispersion is larger in the region of metallic absorption features and thus appears to originate in differences in progenitor composition. To demonstrate this physical explanation conclusively, we used a stationary Monte Carlo radiative transfer code to create a grid of maximum light spectra with varying luminosities and the amount of metals in the outer layers. These model spectra can accurately reproduce the observed dispersion in terms of a varying progenitor composition.

A more detailed PTF-led campaign has now tracked the UV spectra as a function of phase every few days for four selected events. By securing UV spectra immediately after detection we are sensitive not only to the progenitor composition but also to interactions between the SN ejecta and the binary companion. The first results of this effort are particularly promising and may conclusively differentiate various routes to the SN Ia explosion mechanism.

Finally, a new result has followed from the SNLS campaign. Using the fact the SNLS deep fields were imaged repeatedly over 4 years, we have identified and, using Keck, spectroscopicallyconfirmed, 10 Type IIN supernovae with intense Lyman $\alpha$ emission at $\mathrm{z} \sim 2-3$. These represent the most distant SNe so far found. Such SNe IIN are thought to represent core-collapse events from stars with main sequence masses in excess of 40-60 solar masses and so their abundance can provide a guide to the nature of the stellar initial mass function at early cosmic times. In the 
longer term, detailed study of SN IIN with future facilities (TMT, LSST) may provide a valuable probe of the expansion history at redshifts beyond 2 .

\subsection{The Nature and Small-Scale Distribution of Dark Matter}

Individual clusters of galaxies act as excellent 'laboratories' for probing the distribution of dark matter over a wide range of physical scales for comparison with numerical simulations. If dark matter (DM) is a cold non-interacting particle, numerical simulations predict that the DM radial profile should follow the so-called universal Navarro-Frenk-White (NFW) profile. Comparing observations of the DM profile with numerical simulations provides a key test of the gravitational interplay between DM and baryons over cosmic time and offers a fundamental test of the assumption that the DM particle is truly cold.

Through this DOE award we conducted the most rigorous and technically-advanced test of the distribution of DM on small scales. Although earlier cluster analyses apparently supported the NFW profile, those datasets are insufficiently precise and, crucially, do not separate the DM and baryonic components - a key requirement on small scales. In our work we exploit strongly-lensed features of known redshift in the cores of clusters to constrain the small scale DM distribution and weak shear signals to constrain the large scale behavior. Stellar dynamics of the central cluster galaxy enables us to separate the baryonic contribution. For 7 clusters, we have now measured the DM profile over 3 orders of magnitude in physical radius ( $3 \mathrm{kpc}$ to $3 \mathrm{Mpc}$ ) providing a unique test of the NFW profile and its universality. A major technical advance during the period of the award has been demonstration that dynamical data for the halo of the central galaxy and X-ray imaging of the hot gas in the cluster can, with the lensing data, eliminate uncertainties arising from projection effects.

Importantly, we find a much greater dispersion in the inner slope of DM profile than is predicted in the CDM simulations and, for several clusters, a slope much flatter than predicted. At face value this could provide support for a dark matter particle that is 'self-interacting'. Alternatively, it may indicate that the nature of interaction between dark and baryonic matter is poorly understood. Our observed mass distributions thus provide a key basis for improved numerical simulations that attempt to explain the joint behavior of dark and baryonic matter on small scales.

\subsection{Weak Gravitational Lensing as a Probe of Dark Energy}

During the period of the award, we also undertook observational and technical studies of weak gravitational lensing and the degree to which future ground-based facilities (LSST) and space missions (WFIRST, Euclid) may be able to use this phenomenon to provide precise constraints on dark energy.

Weak gravitational lensing utilizes the distorted shapes of thousands of faint background galaxies to infer the foreground distribution of dark matter. Slicing the background population via photometric redshifts enables the growth of structure to be measured; this represents a powerful measure of the balance between gravity and dark energy. Moreover, weak lensing signals can be used to deliver mass-selected cluster samples whose abundance at various redshifts is a further test of dark energy. The merit of counting clusters as a probe of dark energy has been evaluated following completion of a survey spanning $22 \mathrm{deg}^{2}$ undertaken with the Subaru 8-m telescope. $\sim 100$ massive clusters have been located as peaks in the resulting convergence (projected dark matter) maps. The redshifts of these clusters were determined with Keck spectroscopy yielding

halo masses. The complex systematic effects identified in our analysis have clearly defined the challenges this method presents to future ground and space-based cluster surveys. 
Our observational work with the Subaru telescope was paralleled by a technical program to improve algorithms for extracting the weak lensing signals from current and projected future data and to quantify and mitigate systematic errors arising from detector limitations, including nonlinearity, interpixel capacitance, and persistence in the near infrared detectors being considered for future dark energy missions such as WFIRST. The technical studies have been done in conjunction with scientists and engineers at Caltech Optical Observatories, JPL, LBNL and FNAL. Caltech remains very active in the global effort to improve shape measurement algorithms. We maintained a leading role in the Gravitational Lensing Accuracy Test programs (GREAT10), an expanding, worldwide collaboration of groups interested in the technical aspects of weak lensing. Simulated datasets are generated whose weak lensing 'shear' is known a priori. Collaborating groups run independent analysis codes in a blind manner and the results are collated and analyzed. The final workshop of this endeavor, bringing together a significant portion of the active weak lensing community worldwide, was hosted in Pasadena in September 2011.

\section{Refereed Publications from this Award (2009-2012)}

1. The Mean Type Ia Supernova Spectrum over the Past 9 Gigayears, Sullivan, M., Ellis, R.S., Riess, A., Howell, D.A., Nugent, P.E., Gal-Yam, A., Astrophys. J. Lett., 693, L76-80 (2009).

2. The Survival of Dark Matter Halos in the Cluster Cl0024+16, Natarajan, P., Kneib, J-P., Smail, I., Treu, T., Ellis, R.S., Moran, S., Limousin, M. \& Czoske, O., Astrophys. J., 693, 970-983 (2009).

3. Type Ia Supernovae along Redshift: The R(Si II) Ratio and Expansion Velocities in Intermediate Redshift Supernovae, Altavilla, G., Ruiz-Lapuenta, P., Balastegui, A., Mendez, J.,

Irwin, M., Espana-Bonet, C., Ellis, R.S., Folatelli, G., Goobar, A., Hillebrand, W., McMahon, R.G., Nobili, S., Stanishev, V. \& Walton, N.A., Astrophys. J., 695, 135-148 (2009).

4. Subaru Weak Lensing survey - II: Multi-object Spectroscopy and Cluster Masses, Hamana, T., Miyazaki, S., Kashikawa, N., Ellis, R.S., Massey, R.J., Refregier, A. \& Taylor, J.E., Publ. Astron. Soc. J., 61, 833-872 (2009).

5. The Distribution of Dark Matter over 3 Decades in Radius in the Lensing Cluster Abell 611, Newman, A.B., Treu, T., Ellis, R.S., Sand, D.J., Marshall, P.J., Richard, J., Capak, P \& Miyazaki, S., Astrophys. J., 706, 1078-1094 (2009).

6. Pixel-based correction for Charge Transfer Inefficiency in the Hubble Space Telescope Advanced Camera for Surveys, Massey, R.J., Stoughton, C., Leauthaud, A., Rhodes, J., Koekemoer, A., Ellis, R.S. \& Shaghoulian, E., Mon. Not. R. astr. Soc., 401, 371-384 (2010).

7. A Weak Lensing Study of X-Ray Groups in the COSMOS Survey: Form and Evolution of the MassLuminosity Relation, Leauthaud, A., Finoguenov, A., Kneib, J-P., Johnston, D.E., Massey, R.J., Rhodes, J., Taylor, J.E., Capak, P., Ilbert, O., Bundy, K., Cappelluti, N., Ellis, R.S.,Elvis, M., Heymans, C., Koekemoer, A.M., Le Fevre, O., Lilly, S., Mellier, Y., Refregier, A., Salvata, M., Scoville, N., Smoot, G., Tanaka, M., van Waerbeke, L. \& Wolk, M., Astrophys. J., 709, 97-114 (2010).

8. The Effects of Charge Transfer Inefficiency (CTI) on Galaxy Shape Measurements, Rhodes, J., Leauthaud, A., Stoughton, C., Massey, R., Dawson, K., Kolbe, W. \& Roe, N. 2010, Publ. Astron. Soc. Pac., 122, 439-450

9. The Local Cluster Substructure Survey (LoCuSS): First results from strong-lensing analysis of massive clusters, Richard, J., Smith, G.P., Kneib, J-P., Ellis, R.S., Sanderson, A., Pei, L., Targett, T., Sand, D.J., 
Swinbank, M., Dannerbauer, H., Egami, E., Hamilton-Morris, V., Moran, S. \& James, T., Mon. Not. R. astr. Soc., 404, 325-349 (2010)

10. The Type Ia Supernova Rate in Galaxy Clusters at $0.5<z<0.9$, Sharon, K., Gal-Yam, A., Maoz, D., Filippenko, A. V.,Foley, R., Silverman, J., Ebeling, H., Ma, C,-J., Ofek, E.O., Kneib, J.-P., Donahue, M., Ellis, R.S., Freedman, W.L., Kirshner, R.P., Matheson, T.,Mulchaey, J.S., Sarajedini, V.L. \& Voit, M.G., Astrophys. J., 718, 876-893 (2010).

11. The Supernova Legacy Survey 3-year Sample: Type Ia Supernovae photometric distances and cosmological constraints, Guy, J., Conley, A., Sullivan, M., Regnault, N., Astier, P., Balland, C., Carlberg, R.G., Fouchez, D., Hardin, D., Hook, I.M., Howell, D.A. Pain, R., Palanque-Delabrouille, N., Perrett, K.M., Pritchet, C.J., Rich, J., Ruhlmann-Kleider, V., Balam, D., Baumont, S., Ellis. R.S., Fabbro, S., Fakhouri, H.K., Fourmanoit, N., Gonzalez-Gaitan, S., Graham, M.L., Hsiao, E., Kronborg, T., Lidman, C., Mourao, A.M., Perlmutter, S., Ripoche, P., Suzuki, N. \& Walker, E.S., Astron. Astrophys., 523, 7-40 (2010)

12. Supernova Constraints and Systematic Errors from the First 3 Years of the Supernova Legacy Survey, Conley, A., Guy, J., Sullivan, M., Regnault, N., Astier, P., Balland, C., Basa, S., Carlberg, R.G., Fouchez, D., Hardin, D., Hook, I.M., Howell, D.A., Pain, R., Palanque-Delabrouille, M., Perrett, K.M., Pritchet, C.J., Rich, J., Ruhlmann-Kleider, V., Balam, D., Baumont, S., Ellis, R.S., Fabbro, S., Fakhouri, H.K., Fourmanoit, N., Gonzalez-Gaitan, S., Graham, M.L., Hsiao, E., Kronborg, T., Lidman, C., Mourao, A.M., Perlmutter, S., Ripoche, P., Suzuki, N. \& Walker, E.S., Astrophys. J. Suppl., 192, 1-29 (2011).

13. Hubble Space Telescope Studies of Nearby Type Ia Supernovae: The Mean Maximum Light Ultraviolet Spectrum and its Dispersion, Cooke, J., Sullivan, M., Ellis, R.S., Nugent, P., Howell, D.A., Gal-Yam, A., Lidman, C., Bloom, J.S., Cenko, S.B., Kasliwal, M.M., Kulkarni, S.R., Law, N.M., Ofek, E.O. \& Quimby, R.M., Astrophys. J. Lett., 727, L35-38 (2011).

14. The Dark Matter Distribution in Abell 383: Evidence for a Shallow Density Cusp from Improved Lensing, Stellar Kinematic and X-ray Data, Newman, A., Treu, T., Ellis, R.S. \& Sand, D.J, Astrophys. J. Lett., 728, L39-44 (2011)

15. SNLS3: Constraints on Dark Energy Combining the Supernova Legacy Survey Third Year Data with Other Probes, Sullivan, M., Guy, J., Conley, A., Regnault, N., Astier, P., Balland, C., Basa, S., Carlberg, R.G., Fouchez, D., Hardin, D., Hook, I.M., Howell, D.A., Pain, R., Palanque-Delabrouille, N., Perrett, K.M., Pritchet, C.J., Rich, J., Ruhlmann-Kleider, V., Balam, D., Baumont, S., Ellis, R.S., Fabbro, S.,Fakhouri, H.K., Fourmanoit, N., Gonzalez-Gaitan, S., Graham, M.L., Hudson, M.J., Hsiao, E., Kronborg, T., Lidman, C., Mourao, A.M., Neill, J.D., Perlmutter, S., Ripoche, P., Suzuki, N., Walker, E.S., Astrophys. J., 737, 102-120 (2011)

16. PTF10ops - a subluminous, normal-width lightcurve Type Ia supernova in the middle of nowhere, Maguire, K., Sullivan, M., Thomas, R.C., Nugent, P., Howell, D.A., Gal-Yam, A., Arcavi, I., Ben-Ami, S., Blake, S., Botyanski, J., Buton, C., Cooke, J., Ellis, R.S., Hook, I.M., Kasliwal, M.M., Pan, Y-C., Pereira, R., Podsiadlowski, P., Sternberg, A., Suzuki, N., Xu, D., Yaron, O., Bloom, J.S., Cenko, S.B., Kulkarni, S.R., Law, N., Ofek, E.O., Poznanski, D. \& Quimby, R.M., Mon. Not. R. astr. Soc., 418, 747-758 (2011)

17.. Measuring the Geometry of the Universe from Weak Gravitational Lensing behind Galaxy Groups in the HST COSMOS Survey, Taylor, J.E., Massey, R.J., Leauthaud, A., George, M.R., Rhodes, J., Kitching, T.D., Capak, P., Ellis, R.S., Figuenov, A., Ilbert, A., Jullo, E., Kneib, J-P., Koekemoer, A., Scoville, N, Tanaka, M., Astrophys. J., 749, 127- 138 (2012)

\section{Articles Submitted for Publication:}

18. Hubble Space Telescope studies of low redshift Type Ia Supernovae: Evolution with redshift and ultraviolet spectral trends, Maguire, K., Sullivan, M., Ellis, R.S., Nugent, P.E., Howell, D.A., Gal-Yam, A., Cooke, J., Mazzali, P., Pan, Y-C, Dilday, B., Thomas, R.C., Arcavi, I., Ben-Ami, S., Bersier, D., 
Bianco, F.B., Fulton, B.J., Hook, I., Horesh, A., Hsiao, E., James, P.A., Podsiadlowski, P., Walker, E.S., Yaron, O., Kasliwal, M.M., Laher, R.R., Law, N.M., Ofek, E. \& Poznanski, D., Mon. Not. R. astr. Soc., in press (see astro-ph/1205.7040)

19. The Subaru Lensing-Selected Cluster Sample: Further Spectroscopy and Challenges of Using Clusters as Probes of Cosmology, Green, A., Taylor, J., Ellis, R.S., Massey, R.J., Miyazaki, S., Hamana, T., Refregier, A., Astrophys. J., submitted.

20. Spectroscopic Survey of lensed sources and multiply images systems in the field of Abell 1689, Richard, J., Stark, D.P., Kneib, J-P, Mann, A., Smith, G.P., Toft, S., Knudsen, K. K., Ellis, R.S., Limousin, M. \& Egami, E., Mon. Not. R. astr. Soc., submitted.

21. Studying Type Ia Supernova Diversity in the Ultraviolet: Combining Observational Data with Models, Walker, E.S., Hachinger, S., Mazzali, P.A., Ellis, R.S., Sullivan, M., Gal-Yam, A. \& Howell, D.A., Mon. Not. R. astr. Soc., submitted.

22. Rest-frame Ultraviolet Properties of z 2 Type IIn Supernovae, Cooke, J., Sullivan, M., Barton, E., J., Ellis, R.S., Bullock, J.S., Newman, A., O'Meara, J.M. \& Tollerud, E., Astrophys. J., submitted.

23. The X-ray Properties of Weak Lensing Selected Galaxy Clusters, Giles, P.A., Maughan, B.J., Hamana, T., Miyazaki, S., Birkinshaw, M., Ellis, R.S. \& Massey, R., Mon. Not. R. astr. Soc., submitted.

24. The Distribution of Dark Matter in 7 Clusters of Galaxies: The Data, Newman, A., Treu, T., Ellis, R.S., Sand, D.J., Richard, J. \& Jullo, E., Astrophys. J., submitted

25. The Ultraviolet/Optical Spectra of the Type Ia Supernova SN2010jn: A bright supernova with outer layers rich in iron-group elements, Hachinger, S., Mazzali, P.A., Sullivan, M., \& Ellis, R.S., Mon. Not. R. astr. Soc., submitted.

\section{Budget}

A DOE budget page including all funds with explanations.

\section{Personnel Distribution}

Personnel Distribution Table showing present personnel.

\section{Graduate Students}

A summary statement of FTE graduate students and FTE postdocs 